\title{
Real-time processing of event descriptions for partially- and fully-completed events: Evidence from the visual world paradigm
}

\author{
Max Kaplan, Amulya Raju \& Sudha Arunachalam*
}

\begin{abstract}
The current study investigated non-culminating accomplishments through an experimental lens. We used a well-established paradigm for studying real-time language processing using eye-tracking, the visual world paradigm. Our study was modeled after Altmann and Kamide's (2007) investigation of processing of aspectual information contained in a perfect verb form (e.g., has eaten). We compared Englishspeaking adults' interpretations of sentences like 'The girl has eaten a cookie' and 'The girl was eating a cookie' in the context of one of two visual scenes. In the Full Completion condition, the scene depicted two referents that were compatible with the predicate: one was compatible with the expected end state of the event (e.g., an empty plate), the other with an unrealized version of the event (e.g., an uneaten cookie). In the Partial Completion condition, the scene depicted a referent that was compatible with a partially-completed version of the event (e.g., part of a cookie on a plate) and an unrealized interpretation (e.g., an uneaten cookie). For verb forms in the perfect (e.g., has eaten) but not in the progressive, we found a difference between conditions; listeners preferred to look at the fully-affected referent in the Full Completion condition as compared to the partially-affected referent in the Partial Completion condition. We take the results as suggestive in favor of a pragmatic rather than semantic account of non-culmination interpretations in English.
\end{abstract}

Keywords. aspect; events; accomplishment predicates; eye-tracking; culmination

1. Introduction. In a paper famously titled, in part, "A language that can't say, 'I burned it, but it didn't burn' and one that can," Ikegami (1985) presented evidence that in Japanese, perfective accomplishment and achievement predicates (e.g., I burned it) can describe events that have only completed partially. This phenomenon, usually referred to as a non-culminating accomplishment, has been described for typologically unrelated languages including Thai (Koenig \& Muansuwan, 2000), Tamil (Pederson, 2008), Salish languages (e.g., Bar-el et al., 2005), and Hindi (Singh, 1998). Even in English, accomplishment and achievement predicates can sometimes describe events that do not fully culminate. Although \#I burned it, but it didn't burn is odd in English, consider (1a-b). A listener who knows that Infinite Jest is over 500,000 words long will infer that the reader most likely only read part of the novel in (1a) (but finished it in 1b).

What did you do last night?

a. I read Infinite Jest.

b. I read The Cat in the Hat.

\footnotetext{
*We are grateful to the audience at TELIC 2017 (Workshop on Non-culminating, Irresultative and Atelic Readings of Telic Predicates: Combining Theoretical and Experimental Perspectives) in Stuttgart, Germany, and to members of the GRAMALL project (PI: Angeliek van Hout). This research was supported by NSF BCS1748826 to Sudha Arunachalam. Authors: Max Kaplan, University of California (mkaplan2@ucsc.edu), Amulya Raju, Boston University (myamulya@gmail.com), \& Sudha Arunachalam, New York University (sudha@nyu.edu).
} 
Indeed, Arunachalam and Kothari (2011) found in an experimental study that English speakers, like Hindi speakers, often accepted telic predicates as descriptions of events that did not come to their natural culmination point - such as "She closed the door" to describe a door left ajar, or "She ate the cookie" to describe a partially-eaten cookie. In this experiment, Englishspeaking adults and Hindi-speaking adults watched videos of events performed by a human actor. In the cookie-eating video, for example, the actor picked up a cookie, ate approximately half of it, and put the remaining half onto the plate, where it remained until the end of the video. Although the event culminated, it did so at an odd, premature point given how eating events typically unfold. In Hindi, such events are describable with a simple perfective construction (Singh, 1998), and Hindi speakers in the experiment accepted such descriptions for these partially-completed events about half the time (53\%) (as contrasted with a serial verb construction which does not permit, or only rarely permits, partial completion interpretations). Surprisingly, Arunachalam and Kothari found that English speakers too accepted descriptions in the simple past for these partially-completed events $47 \%$ of the time, nearly the same rate (see also Patt, Wagner, \& Arunachalam, 2020 for results from a graded acceptability judgment task).

But while these experimental results reveal the acceptability judgments that speakers ultimately arrived at for these event descriptions, they do not reveal the processes underlying these judgments. Experimental studies on the time-course of processing have shown that listeners use aspectual information in real time to guide their interpretation of event descriptions (e.g., Altmann \& Kamide, 2007; Bott \& Gattnar, 2015; Foppolo et al., 2018). For example, Altmann and Kamide (2007), using a visual world eye-tracking paradigm, found that English speakers who heard sentences in the perfect form (e.g., The woman has eaten...) preferred to look at a picture of an empty plate as compared to a picture of an uneaten cake on a plate when the verb was uttered, suggesting that they quickly computed the aspectual information in the verbal form and looked to the picture compatible with a completed event. Given that English speakers also accept partial completion interpretations for telic predicates, we can ask whether they, in real time, entertain partial completion interpretations when the verb is uttered. ${ }^{1}$ If so, this would suggest that partial completion interpretations are compatible with the aspectual information available in the English perfect verbal form. If not, it would suggest that listeners can ultimately accept partial completion interpretations, but that doing so requires extra processing.

To explore this issue, we conducted an experiment designed to ask whether, and if so, when, English speakers permit non-culminating construals of event descriptions. We adapted Altmann and Kamide's (2007) paradigm, but instead of depicting an empty plate and an untouched cake as the possible referents, we depicted either a completely-eaten cake (i.e., empty plate) or a partially-eaten cake alongside a whole untouched cookie (the non-target referent). Our logic was as follows: If English speakers find descriptions in the perfect form (e.g., has eaten) to be appropriate for non-culminating as well as culminating events, they should show just as strong a preference to look at referents of partially-completed events as referents of fully-completed events; given that the other relevant object in the scene is a completely unaffected referent (whole cake), the partially-affected referent is the best referent given the visual scene. In Altmann and Kamide's study, even before the onset of the determiner in the direct object - that is, during the time window including the auxiliary/modal and main verb-participants looked more

\footnotetext{
${ }^{1}$ We acknowledge that there is a difference between the English simple past and perfect form. The simple past was used in examples (1a) and (1b) and Arunachalam and Kothari (2011), but we chose the perfect form to permit close alignment with Altmann and Kamide (2007).
} 
to the empty plate in the perfect condition than in the future condition; therefore, we too expected effects of aspectual processing to appear during the perfect form, before the direct object.

Like Altmann and Kamide, we presented participants with descriptions of events in the perfect and evaluated their gaze to visual scenes depicting the direct object referents of either a fully-completed version (Full Completion condition) or a partially-completed version (Partial Completion condition) of the event, as well as a depiction of the direct object referent in untouched form - before the event occurred. In a second (control) condition, participants saw exactly the same visual stimuli, again with some trials in the Full Completion condition and others in the Partial Completion condition, but the sentences used the past progressive form (e.g., was eating). For each aspectual condition, we examined preference for the partially- or fully-affected referent, specifically during the time period from the onset of the verb to the onset of the determiner in the phrase labeling the object. In the visual world paradigm (Allopenna, Tanenhaus, \& Magnuson, 1998), listeners look to upcoming referents at the earliest opportunity; their gaze patterns are influenced not only by the auditory stream but also by how closely each visual image on the screen matches that auditory stream. Thus, listeners' gaze patterns reflect how well the partially- or fully-affected referent is taken as the best match for the unfolding utterance.

With respect to the descriptions in the perfect, if speakers are happy to accept partially-completed events as their referents, then we expect no significant difference between their gaze to the fully-affected referent in the Full Completion condition and the partially-affected referent in the Partial Completion condition. This outcome would indicate that listeners entertain a partiallycompleted interpretation as part of their initial processing of the verb form, rather than accepting such an interpretation only later in processing. If, on the other hand, listeners have difficulty entertaining this possibility, even though it is the best match for the unfolding sentence, they should look to the fully-affected referent in the Full Completion condition significantly more than the partially-affected referent in the Partial Completion condition during the verb, and only later, perhaps after the sentence is complete, show equal preference for both event referents.

With respect to the progressive descriptions, the grammatical properties of the utterance itself do not lead to strong predictions about listeners' gaze patterns. It may be that listeners most easily entertain an "interrupted" event interpretation of the utterance as it unfolds, in which case we would expect that they would prefer the partially-affected referent in the Partial Completion condition as compared to the fully-affected referent in the Full Completion condition. But some research has suggested that listeners do not do so (Madden \& Zwaan, 2003), instead considering both partially- and fully-completed referents as equally appropriate for event descriptions with progressive verb forms. Nevertheless, the progressive condition can serve as a control, because under no circumstance do we predict listeners to prefer partially-affected referents less than fully-affected referents, as we do for the perfect verb form under one hypothesis.

The immediate goal of this experiment was to determine whether English speakers entertain partial completion interpretations when processing a verb form in the perfect. However, the outcome may also shed light on the nature of non-culminating accomplishments. Theoretical accounts of non-culminating accomplishments have typically focused on either semantic or pragmatic issues (see, e.g., Gyarmathy \& Altshuler, for discussion). On a semantic account, the semantics of the verb form itself permit partial completion interpretations (e.g., Singh, 1998); a pragmatic account focuses on a cancellable implicature that the event comes to full completion. Experimental evidence can shed light on this issue. Arunachalam and Kothari (2011) took their experimental results as evidence for a pragmatic account for Hindi, in part because English showed a similar pattern of judgments to Hindi despite differences in how perfective aspect is 
expressed in the two languages. Here, we suggest that experimental evidence from real-time sentence processing can be particularly helpful: If the semantics of the verb form allow a nonculminating event construal, then we would expect that such computation could occur incrementally, during the verb phrase. If the evidence suggests that a non-culminating event construal only becomes available after the sentence is complete, this would be less compatible with a semantic account, and perhaps more compatible with a pragmatic account in which calculation or cancelation of implicatures may occur after the fact. Pragmatic information can influence online processing, but sometimes is integrated only later (Huang \& Snedeker, 2009, 2018).

The inferences we can draw from experimental evidence are, however, limited. Pragmatic information, if available, can sometimes influence processing early on, and can even constrain the hypotheses that a listener or reader makes about how the sentence will unfold or what its interpretation will be (e.g., Kaiser \& Trueswell, 2004). Therefore, even if a particular result obtains during incremental processing before the utterance is complete, a pragmatic explanation cannot be ruled out. It is also the case that if multiple semantic interpretations are possible, a listener or reader might initially only compute the one that is most salient or most frequent in their experience. Therefore, in our design, we make the non-culmination interpretation salient and plausible by depicting the partially-affected referent in the visual scene. Further, we acknowledge that we can only interpret our results if participants do not appear to compute the non-culmination interpretation until after the verb phrase is fully complete - that is, not in an incremental fashion.

\section{Method}

2.1. PARTICIPANTS. Fifty-two adults ( 9 male, 43 female, mean age 21 years) participated. All self-reported as monolingual native English speakers with normal or corrected-to-normal vision and hearing. They received payment at a rate of $\$ 14 / \mathrm{hr}$.

2.2. Design. To determine whether native English-speaking listeners entertain partial completion interpretations of perfect and progressive verb forms as the utterances unfold to the same extent as they do full completion interpretations, we adapted Altmann and Kamide's (2007) design using the visual world paradigm. We employed a 2 x 2 design, with aspect (perfect vs. progressive) manipulated between-subject (participants were randomly assigned to the perfect or progressive condition), and event completion (partial vs. full) manipulated within-subject. We chose this design because pilot testing for another similar study suggested that participants noticed that there were two sentence types and tried to contrast them; we wanted to avoid this and so made this manipulation between-subject.

Participants viewed eight experimental trials in a fixed order. Each began with an introduction to a character; a picture of a person or animal appeared in the center of the screen accompanied by an auditory carrier frame naming the character and introducing the trial (e.g., This one is about a girl). Then, they viewed the target scene, which depicted four images (Figure 1). Two of the images were of the same referent in varying stages of affectedness and the other two were unrelated distractor images. The accompanying sentence conveyed an event description that related to one of the pictures (e.g., The girl has eaten the cookie).

In the Full Completion condition, the two images depicted a referent that indicated the event had not taken place at all — a whole cookie on a plate - and a referent that indicated the event had completed to its natural culmination point - a plate with a few cookie crumbs on it. (We included the crumbs to suggest that a cookie had indeed been there at some point. Of course, not all of the predicates required that full culmination be depicted by an absent referent.) This condition was parallel to the one used by Altmann and Kamide (2007). 
a)

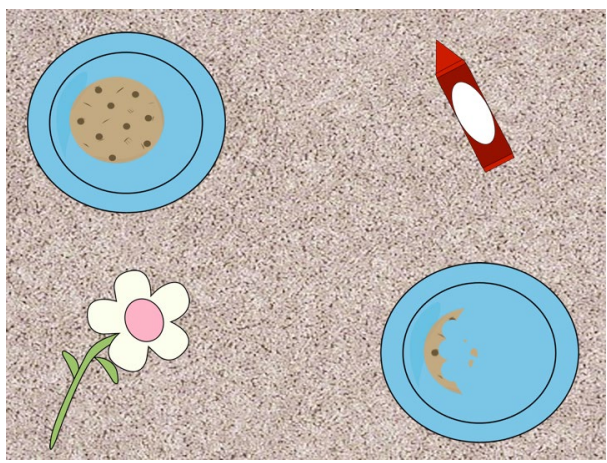

b)

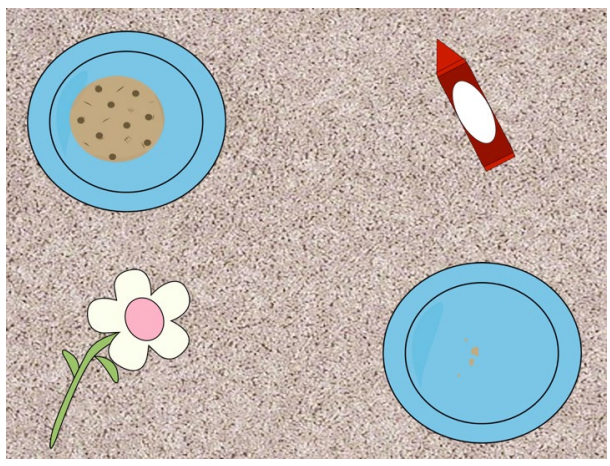

Figure 1. Visual stimuli for one experimental trial (has eaten/was eating the cookie), in (a) the Partial Completion condition, and (b) the Full Completion condition.

In the Partial Completion condition, the first image was identical-whole cookie on a plate - and the other depicted a partially-affected referent - part of a cookie on a plate. The partially-affected referents depicted the event as approximately $75 \%$ complete. This is similar to most other work on this topic except some recent studies (e.g., van Hout et al., 2017).

We refer to the fully- or partially-affected referent (e.g., plate with crumbs or partially-eaten cookie in the Full Completion and Partial Completion conditions, respectively) as the target image, and the unaffected referent (e.g., whole cookie) as the competitor image because it depicts an object of the same basic level category as the target but is not the best referent given the utterance. The two distractor images were implausible referents given the verb (e.g., were not edible).

One notable feature of our design is that in both the Partial and Full Completion conditions the target referent is either partially or fully affected, and the competitor is not affected at all. We did not pit the partially- and fully-affected referents against each other on the same trial. Naturally we expected that given both partially- and fully-completed event referents in the same display to choose from, listeners would prefer the fully-completed event referent; indeed, recent evidence from Foppolo et al. (2018) in a similar design suggests that they would. Our design choice was intended to "stack the deck" against finding differences between the conditions: Whether or not the partially-completed event referent is the ideal interpretation of the unfolding utterance, it is the only one that is compatible with the visual scene given the simple SVO structure of the sentences heard in the experiment (and barring less likely continuations like "has eaten none of the cookie"). If participants do not strongly and immediately consider the partial completion interpretation, they may look back and forth at the two cookie-related images, because both are associated with eating. Thus, we expect participants' gaze to be affected by their relative preference for the two referents on the screen that are most related to the event being described - the target (whether partially or fully affected) and the unaffected competitor object.

Participants also viewed one warm-up trial at the beginning of the experiment that was designed to expose them to the trial structure, as well as 10 filler trials interleaved with the experimental trials. The warm-up and filler trials did not involve perfect or progressive forms and did not depict referents affected by different degrees of event culmination. Eight of the filler trials did depict two referents that formed a pair (e.g., a red hat and a blue hat; a peanut butter and jelly sandwich and a cheese sandwich); on 5 of these one of these referents was the target and on the other 3 it was not. We thus included 5 filler trials on which the target was a member of a pair and 5 on which it was not; this was to ensure that participants did not expect that one member of a pair would always be the referent being described. 
2.3. MATERIALS. Visual stimuli consisted of freely available clip-art images, digitally edited and placed on a neutral background (these same stimuli were used by Patt et al., 2020). The auditory stimuli were recorded by a female native speaker of American English, and were uttered with slow prosody, with a mean utterance duration of 1900 ms. As in Altmann and Kamide (2007), the critical sentences began exactly $1000 \mathrm{~ms}$ after the four images appeared on the screen on each trial; this allowed participants to first view the scene in silence. The onsets and offsets of the critical points in the sentence were identified for analysis using Praat (Boersma \& Weenink, 2005). A description of the stimuli for all experimental trials is in Table 1.

\begin{tabular}{|c|c|c|c|}
\hline \multirow[t]{2}{*}{ Sentence } & \multicolumn{2}{|l|}{ Target } & \multirow[t]{2}{*}{$\begin{array}{l}\text { Competitor } \\
\text { (Unaffected Object) }\end{array}$} \\
\hline & $\begin{array}{l}\text { Partial Comple- } \\
\text { tion Condition }\end{array}$ & $\begin{array}{l}\text { Full Completion } \\
\text { Condition }\end{array}$ & \\
\hline $\begin{array}{l}\text { The girl has eaten/was eat- } \\
\text { ing the cookie. }\end{array}$ & $\begin{array}{l}\text { Partially-eaten } \\
\text { cookie on a plate }\end{array}$ & Plate with crumbs & Full, uneaten cookie \\
\hline $\begin{array}{l}\text { The boy has covered/was } \\
\text { covering the pot. }\end{array}$ & Pot with lid askew & $\begin{array}{l}\text { Pot fully covered } \\
\text { by lid }\end{array}$ & $\begin{array}{l}\text { Uncovered pot next } \\
\text { to its lid }\end{array}$ \\
\hline $\begin{array}{l}\text { The baby has drunk/was } \\
\text { drinking the milk. }\end{array}$ & $\begin{array}{l}\text { Baby bottle half- } \\
\text { filled with milk }\end{array}$ & Empty baby bottle & $\begin{array}{l}\text { Baby bottle filled } \\
\text { with milk }\end{array}$ \\
\hline $\begin{array}{l}\text { The lady has closed/was } \\
\text { closing the box. }\end{array}$ & $\begin{array}{l}\text { Half-closed card- } \\
\text { board box }\end{array}$ & $\begin{array}{l}\text { Closed cardboard } \\
\text { box }\end{array}$ & Open cardboard box \\
\hline $\begin{array}{l}\text { The teacher has drawn/was } \\
\text { drawing a circle. }\end{array}$ & $\begin{array}{l}\text { Partially-drawn cir- } \\
\text { cle with crayon }\end{array}$ & $\begin{array}{l}\text { Fully-drawn circle } \\
\text { with crayon }\end{array}$ & $\begin{array}{l}\text { Crayon without any- } \\
\text { thing drawn }\end{array}$ \\
\hline $\begin{array}{l}\text { The doctor has opened/was } \\
\text { opening the book. }\end{array}$ & $\begin{array}{l}\text { Partially-opened } \\
\text { book }\end{array}$ & Open book & Closed book \\
\hline $\begin{array}{l}\text { The kid has peeled/was } \\
\text { peeling the banana. }\end{array}$ & $\begin{array}{l}\text { Partially-peeled ba- } \\
\text { nana }\end{array}$ & $\begin{array}{l}\text { Peeled banana next } \\
\text { to its peel }\end{array}$ & Unpeeled banana \\
\hline $\begin{array}{l}\text { The man has filled/was fill- } \\
\text { ing the glass. }\end{array}$ & $\begin{array}{l}\text { Glass partially } \\
\text { filled with marbles }\end{array}$ & $\begin{array}{l}\text { Glass fully filled } \\
\text { with marbles }\end{array}$ & Empty glass \\
\hline
\end{tabular}

Table 1. Auditory stimuli and description of target and competitor objects depicted on all trials.

2.4. ApPaRATUS. Stimuli were presented on a 24" widescreen Tobii T60XL eye tracking monitor, which samples binocularly at 60 frames/second. The auditory stimuli played via integrated stereo speakers in the monitor.

2.5. Procedure. Participants sat in front of the eye tracking monitor, with their eyes between 60 and $70 \mathrm{~mm}$ from the display. Calibration took approximately $20 \mathrm{~s}$, after which time participants were simply asked to watch the video; they were not given an explicit task. Although many studies using the visual world paradigm require the listener to complete a task (e.g., moving physical objects, answering questions), Altmann and Kamide (2007) found the effects we have described with passive viewing. The entire experiment took less than $5 \mathrm{~min}$.

3. Coding and analysis. From the raw data output, we first determined where participants' gaze was directed on each frame: target image, competitor image, unrelated distractor images, elsewhere (e.g., in the middle of the four images or off-screen), or track loss (e.g., blinks). We planned to exclude participants with greater than $35 \%$ track loss over the experimental session, but none met this criterion; the average percentage of track loss was $10 \%$ for participants in the perfect condition and $8 \%$ for participants in the progressive condition. 
Next, we identified two windows of analysis for each trial. We identified for each trial the onsets and offsets of the auxiliary/modal, the DP labeling the direct object, and the offset of the utterance. For the first window of analysis, we assessed gaze from each trial's onset of the auxiliary/modal to the onset of the DP labeling the direct object. We refer to this as the Verb window, whose duration averaged $1 \mathrm{~s}$. This is the window during which online processing of the verb should affect gaze. For the second window of analysis, we assessed gaze for a period of the same duration ( $1 \mathrm{~s}$ ) beginning from the offset of the utterance. We anticipated that gaze behavior during this period would reflect participants' ultimate interpretation of the utterance (and that this should accord with offline judgments). We refer to this as the Post-Utterance window.

We analyzed the perfect and progressive conditions separately. Our goal was to compare, within each condition, whether participants showed a difference in their gaze to the target image according to whether the images depicted Partial or Full completion. Therefore, our primary dependent measure was looks to the target image across conditions; this was a binary variable with a value of 1 for each frame on which participants looked to the target image, and a value of 0 for each frame on which participants did not. We aggregated these data into $\sim 50 \mathrm{~ms}$ bins of three frames each, and then transformed them using an empirical logit function to reduce the effects of eye-movement based dependencies (the eyes cannot shift from one scene to another in a single frame) (Barr, 2008). We fit the data using a multi-level logistic regression model using the lmer() function in the lme4 package (Bates, Maechler, Bolker, \& Walker, 2015) in R version 3.3.0 (R Core Team, 2013). For each aspectual condition, the model included random effects of Participant and Trial and fixed effects of Condition (Partial vs. Full) and Time (in seconds).

As Altmann and Kamide (2007) pointed out, if this analysis reveals a difference between the Partial and Full Completion conditions there are two possible interpretations. One is our intended interpretation, that participants are affected by the relative fitness of the target and competitor as referents for the event description. However, an alternate possibility is that the difference between conditions is unrelated to the competitor-perhaps participants look differently to the unrelated distractor images in each condition. Therefore, to ensure that the results of our primary analyses are due to gaze patterns to the target and competitor relative to each other, as opposed to other areas, we included a secondary analysis also included by Altmann and Kamide: we combined looks to the target and competitor, and again asked if there was a difference between conditions. If, as we predict, there is no difference, it would indicate that any patterns in the initial analysis reflect a difference due to relative preference for the target and competitor.

4. Results. Participants' gaze data is depicted in Figures 2 (Perfect condition) and 3 (Progressive condition). In representing the data visually, we plotted the "target advantage", or preference for the target image relative to preference for the competitor, by subtracting the value for the competitor from the value for the target at each frame. Thus, values above 0 reflect a preference for the target as compared to the competitor, values below 0 reflect a preference for the competitor as compared to the target, and values of 0 reflect no preference. This metric allows us to visually depict participants' gaze to the target in relation to their gaze to the competitor. The $\mathrm{x}$-axis depicts time in seconds from when the visual scene appeared on the screen; recall that the auditory stimuli began 1 second later. The figure delineates two time windows, one (Verb window) including the auxiliary and verb until the onset of the determiner in the direct object and lasting on average one second, and the other (Post-Utterance window) including one second beginning at the offset of the entire utterance. For visual inspection, the windows are depicted using the mean values of the onsets and offsets across all utterances, but the statistical analyses used time windows that were adjusted for the onsets and offsets of each utterance/trial independently. 
4.1. PERFECT CONDITION. Inspection of Figure 2 reveals that participants who heard utterances in the perfect form (e.g., "has eaten") indeed showed a difference between trials on which they saw a fully- vs. a partially-affected referent. During the Verb window, participants showed a greater target advantage on Full completion trials than Partial completion trials. We infer that as the sentence unfolded, during the point at which aspectual information was heard, fully-affected referents are taken as better referents of the event description than partially-affected referents. This is true despite that in the visual scene, the competitor image was unaffected by the event and therefore the partially-affected referent was the best candidate available.

If this result carried over throughout participants' processing of the sentence, we would infer that adults do not accept partial completion interpretations of such sentences. However, at approximately $3.5 \mathrm{sec}$, the offset of the entire utterance, participants showed a spike in target advantage on Partial completion trials. It appears that participants engaged in revision or postprocessing that led them to the partially-affected referent as the best candidate given the display. By the end of the 6-sec period during which the scene was on the screen, we see no difference between conditions, suggesting that participants ultimately converged on the partially-affected referent (on Partial completion trials) or the fully-affected referent (on Full completion trials).

Statistical analysis of these patterns revealed that during the Verb window, there was, as predicted, a significant effect of condition (Partial vs. Full). See Table 2. There was also a significant interaction between Time and Condition, reflecting the fact that over the course of the time window, the Partial Completion condition showed a negative spike while gaze in the Full Completion condition remained steady. In the Post-Utterance window, however, encompassing a 1 -second window from utterance offset, we found no effect of condition and no interaction.

In Figure 2, the difference between conditions appears to begin even before the Verb window. Such a difference could not plausibly be attributed to the linguistic stimuli. Therefore, in a post-hoc analysis we evaluated gaze during the approximately $600 \mathrm{~ms}$ after the utterance began but before onset of the auxiliary. This regression analysis revealed that the apparent difference in the pre-verb window is not statistically significant $(p=0.64)$. Trial-by-trial post-hoc analyses indicate that the trend is driven by the last four trials and not the first four; despite interleaved fillers, it is likely that participants could anticipate the sentence type as the session proceeded.

For the secondary analysis combining looks to target and competitor, we found no significant effect of condition in either the Verb or Post-Utterance window. Thus, the significant effect of Condition in the Verb window in the previous analysis should be seen as a result of the influence of the competitor image. That is, during the Verb window, participants in the Partial Completion condition entertained the possibility that the unaffected competitor image was the best referent to a greater extent than participants in the Full Completion condition. 


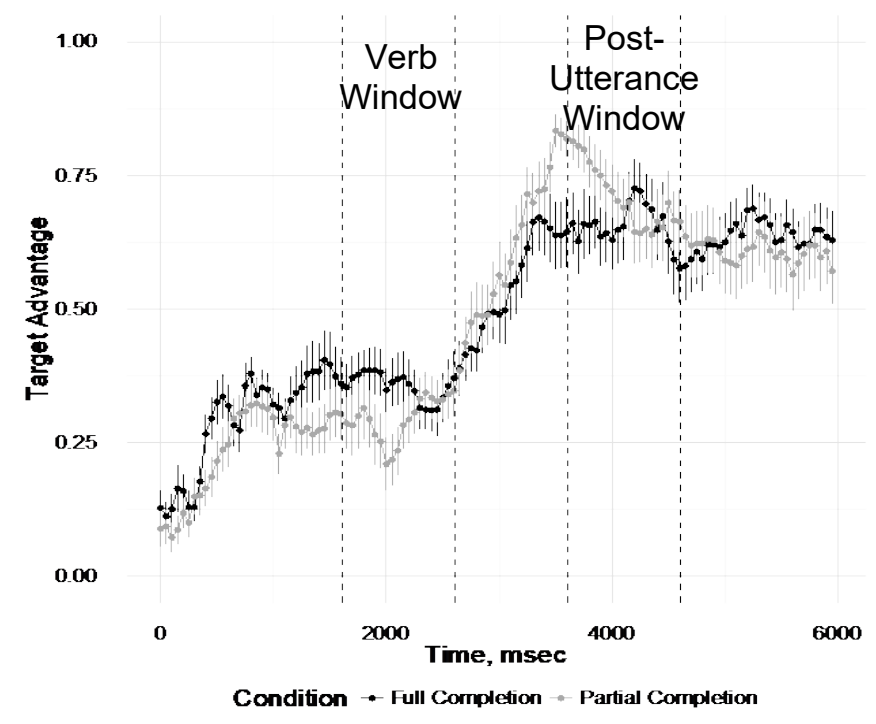

Figure 2. Target advantage (looks to the target minus looks to the competitor) over time in the perfect condition. The left two dotted lines indicate the boundaries of the Verb time window and the right two indicate the Post-Utterance time window (averaged across trials).

Verb window

Looking to target as $\mathrm{dv} \quad$ Looking to target or competitor as

$\mathrm{dv}$

\begin{tabular}{lllllll}
\hline & Estimate & $\mathrm{SE}$ & $t$-value & Estimate & $\mathrm{SE}$ & $t$-value \\
\hline Intercept & 1.53 & 0.058 & 26.28 & 1.66 & 0.074 & 22.51 \\
\hline Condition & $0.26^{*}$ & 0.059 & 4.47 & 0.24 & 0.13 & 1.83 \\
\hline Time (sec) & 0.061 & 0.046 & 1.33 & 0.043 & 0.032 & 1.34 \\
\hline $\begin{array}{l}\text { Condition } \\
\text { x Time in- } \\
\text { teraction }\end{array}$ & -0.28 & 0.088 & -3.17 & -0.093 & 0.062 & -1.51 \\
\hline
\end{tabular}

Post-utterance window

Looking to target as dv

Looking to target or competitor as

dv

\begin{tabular}{lllllll}
\hline & Estimate & SE & $t$-value & Estimate & SE & $t$-value \\
\hline Intercept & 1.82 & 0.11 & 17.08 & 1.87 & 0.11 & 17.80 \\
\hline Condition & -0.29 & 0.19 & -1.51 & -0.24 & 0.20 & -1.22 \\
\hline Time (sec) & -0.0069 & 0.025 & -0.27 & -0.0099 & 0.025 & -0.39 \\
\hline Condition & 0.070 & 0.048 & 1.47 & 0.055 & 0.048 & 1.15
\end{tabular}

$\mathrm{x}$ Time in-

teraction

*model comparison indicates that the parameter contributes significantly at an alpha level of .05

Table 2. Parameter estimates for logistic regression models for the perfect condition

4.2. Progressive CONDition. The progressive condition (e.g., was eating), whose results are depicted in Figure 3, shows a different pattern. Participants showed no difference in target advantage for Partial vs. Full completion trials during the Verb window. There is a trend toward 
greater target advantage in the Partial Completion condition in the Post-Utterance window and beyond, as we might expect if participants tend to favor an ongoing interpretation for the progressive form, but it is small. There is also no evidence of an early target preference in this condition, which indicates that the apparent — though not statistically significant — early preference in the perfect condition was due to the linguistic stimulus heard over the course of the experiment and not simply to features of the visual stimulus.

Statistical analyses confirm these patterns. See Table 3 . We found no effect of condition in either window, and again, the control analyses collapsing target and competitor into a single dependent variable also showed no effect in either window. The trend toward a greater preference for the target in the Partial Completion as compared to the Full Completion condition during the Post-Utterance window evinces in the direction of the parameter estimate for Condition in this analysis, given that Condition was coded with Partial Completion as -0.5 and Full Completion as 0.5 . However, this parameter did not contribute significantly, indicating that there was no reliable difference between conditions.

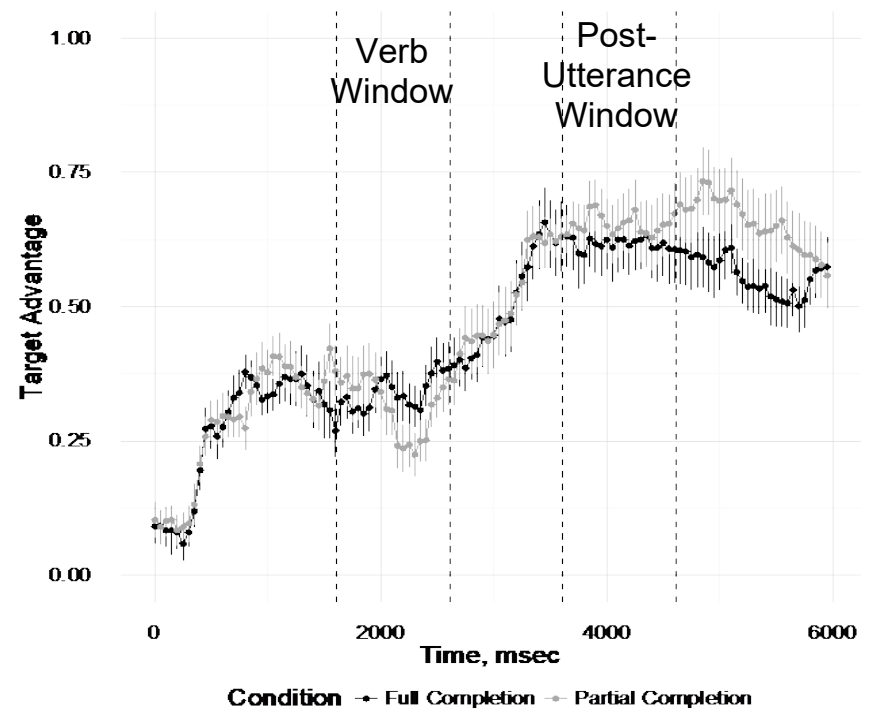

Figure 3. Target advantage (looks to the target minus looks to the competitor) over time in the progressive condition. The left two dotted lines indicate the boundaries of the Verb time window and the right two indicate the Post-Utterance time window (averaged across trials).

\section{Verb window}

Looking to target as dv

Looking to target or competitor as dv

\begin{tabular}{lllllll}
\hline & Estimate & SE & $t$-value & Estimate & SE & $t$-value \\
\hline Intercept & 1.64 & 0.072 & 22.81 & 1.84 & 0.091 & 20.07 \\
\hline Condition & -0.13 & 0.067 & -1.93 & -0.21 & 0.17 & -1.19 \\
\hline Time (sec) & -0.16 & 0.062 & -2.61 & -0.039 & 0.044 & -0.088 \\
\hline $\begin{array}{l}\text { Condition } \\
\text { X Time in- } \\
\text { teraction }\end{array}$ & 0.26 & 0.12 & 2.21 & 0.11 & 0.086 & 1.23 \\
& & & & & & \\
\end{tabular}


Post-utterance window

\begin{tabular}{lllllll}
\hline & Looking to target as $\mathrm{dv}$ & \multicolumn{3}{l}{ Looking to target or competitor as dv } \\
\hline & Estimate & $\mathrm{SE}$ & $t$-value & Estimate & $\mathrm{SE}$ & $t$-value \\
\hline Intercept & 1.75 & 0.11 & 15.63 & 1.86 & 0.072 & 26.01 \\
\hline Condition & -0.096 & 0.21 & -0.46 & -0.010 & 0.14 & -0.071 \\
\hline Time (sec) & 0.0059 & 0.030 & 0.20 & -0.0042 & 0.019 & -0.22 \\
\hline $\begin{array}{l}\text { Condition } \\
\text { X Time in- } \\
\text { teraction }\end{array}$ & 0.021 & 0.058 & 0.36 & 0.0013 & 0.039 & 0.032 \\
\hline
\end{tabular}

Table 3. Parameter estimates for logistic regression models for the progressive condition.

5. Discussion. The results of this study contribute to our understanding of how aspectual information is integrated during online processing, specifically with respect to partial culmination readings. We pursued a finding from Altmann and Kamide (2007) that listeners expect verbs in perfect forms (e.g., has eaten) to refer to fully completed events rather than events that have not yet occurred. Our question was whether this finding would extend to event referents that had only partially completed, rather than fully completed. This investigation was inspired by the fact that English speakers sometimes accept partial completion interpretations of telic predicates with perfect verb forms - although perhaps to a lesser extent than speakers of certain other languages. We investigated whether these partial culmination interpretations arise online, even as the verb form unfolds, or whether they are arrived at later in processing when it is clear that there are no other (better) interpretations available given the context; this work therefore has implications for understanding non-culminating accomplishments in English and other languages.

We used a task similar to Altmann and Kamide's, except that on each trial, participants viewed the outcome of either a fully-completed event (e.g., a plate with crumbs on it) or a partially-completed event (e.g., the remains of a partially eaten cookie on a plate), with a pristine unaffected object as the critical distractor (e.g., a whole cookie on a plate). They heard either perfect forms (e.g., has eaten) or progressive forms (e.g., was eating).

For perfect forms, a difference between completion types surfaced during the verb; participants looked more at the fully-affected referent in the full completion condition than they looked at the partially-affected referent in the partial completion condition during the time window encompassing the auxiliary and verb. Although the partial completion interpretation was the best one given the visual scene, participants did not immediately entertain this interpretation as strongly as they did the fully-affected referent in the Full condition. Then, toward the end of the utterance, participants appeared to reevaluate their initial interpretation and settle on the partially-affected referent. From one second after the offset of the utterance, there was no difference between the partial and full completion conditions, indicating that ultimately, participants arrived at the intended interpretation in both. The finding that at the end of the sentence, participants converged on the partially- and fully-affected referents to the same extent, aligns with Arunachalam and Kothari's (2011) results in an offline judgment task for sentences in the simple pastEnglish speakers do ultimately accept partial completion interpretations of telic predicates at a relatively high rate.

For progressive forms, which served as a control, participants showed no difference in gaze patterns between trials on which the affected referent was partially affected and when it was fully affected, suggesting that they did not have a strong preference for either partial or full completion interpretations for the unfolding utterance. This lack of difference between completion types 
surfaced both during the verb form itself, as well as in a time window of comparable length after the utterance was completed. We interpret this null result cautiously, however, as we only had a small number of items and we may not have had sufficient power to detect a small effect.

We suggest that these findings indicate that arriving at a partial completion interpretation for a perfect form requires some kind of extra calculation: If listeners examined the visual scene and noted that only one referent was compatible with a description using the perfect form (because the competitor object was not at all affected), they should have been able to compute the partial completion interpretation online, as the sentence unfolded. What kind of calculation might this be, and why might it require extra time? We have suggested that although pragmatic information can be integrated incrementally and influence processing in real-time, it is more likely that an after-the-fact calculation would be a pragmatic one rather than a semantic one, particularly for English speakers for whom non-culminating accomplishments are relatively rare.

Therefore, we suggest that although these experimental results cannot by themselves be taken as evidence for a pragmatic account over a semantic account of non-culminating accomplishments, to the extent that this processing is delayed, it is more likely to be compatible with a pragmatic than a semantic explanation. Several studies suggest that aspectual coercion takes time (e.g., Piñango, Winnick, Ullah, \& Zurif, 2006; Todorova, Straub, Badecker, \& Frank, 2000), and accepting non-culminating interpretations for verb forms in the perfect may require similar kinds of processing. It could also be that the correct account is a semantic one, but one in which there is a certain 'looseness' permitted in the interpretation that is assigned (Lasersohn, 1999).

Of course, these findings for English may not carry over to languages in which non-culmination interpretations are easier to arrive at, and for which particular grammatical constructions are associated more strongly with these interpretations than others. In languages like Hindi, listeners may find it easier to entertain partial completion interpretations for single verbs in perfective form, and may do so early in processing. For Hindi, this could be, for example, because their language uses a different grammatical construction, the serial verb construction, to unambiguously signal full culmination, or because the semantics of the perfective are inherently different and permit partial completion interpretations. We have reason to expect strong differences in processing that are attributable to cross-linguistic differences in how completion is grammatically encoded (e.g., Flecken, Stutterheim, \& Carroll, 2014; Sakarias \& Flecken, 2017). Future work investigating how these processes unfold in other languages may shed light on whether any cross-linguistic differences are semantic or pragmatic.

Another consideration for future work is how affected the affected referents must be. In our experiment, the partially-affected referent appeared as though the event was at least $50 \%$ complete, and in most cases approximately $75 \%$ complete. Would even less affected referents show a different pattern, perhaps a difference between the partial and full completion conditions even at the end of the utterance? Conversely, would more affected referents (e.g., reflecting 90\% completion) show no difference between conditions even during the verb? The choice of sentences may matter as well; although the phenomenon of non-culminating accomplishments is typically illustrated by canceling the expected culmination (e.g., .. but not completely), we opted not to study processing of cancelations, instead depicting the possibility of cancelation in the visual stimuli. Adding an explicit cancelation to the sentence might, however, reveal an interesting processing timecourse as listeners make and cancel the culmination implicature.

A second consideration is the particular verbs/predicates that are chosen. We chose predicates that were used in similar prior work and that could be clearly depicted in the simple images we used for the experimental stimuli. However, verbs differ in how likely they are to yield telic 
and atelic interpretations. The definiteness of the verb's object plays a role as well (e.g., Ogiela, Schmitt, \& Casby, 2014). We used only definite determiners for the current stimuli, except with the verb of creation draw (has drawn / was drawing a circle). Further work in this vein can examine how features of the predicate (such as the lexical semantics of the verb) affect not only telicity also partial vs. full completion interpretations.

This work increases our understanding of what kinds of information comprehenders have access to and make use of online, as linguistic information unfolds. Comprehension involves creating a mapping between the unfolding utterance and a mental representation of an event as it is expected to take place in the real world (see Altmann \& Mirković, 2009; McRae \& Matsuki, 2009 for reviews). For example, Bicknell et al. (2010) found that English-speakers hearing a SVO sentence integrate the agent and verb and make predictions about the upcoming patient based on their real-world knowledge about plausible events, and Altmann and Kamide (1999) found that comprehenders use a verb's semantic properties to predict what kind of direct object will be heard next. The study we most closely followed on, Altmann and Kamide (2007), broadened these findings to say that aspects of the event representation like future events and states are also accessible to the parser online, as the verb phrase unfolds (see also Altmann \& Kamide, 2009). Our study is the only one of these to claim that listeners do not always predict an upcoming referent with equal facility, and in doing so it identifies a limitation on what interpretations listeners are able to entertain as tense and aspectual information unfold, specifically related to the extent to which the event comes to its natural culmination point.

In ongoing work, we are examining the developmental origins of event culmination interpretations (Arunachalam, 2017; He \& Arunachalam, 2017). In infancy, there are tight links between linguistic knowledge and conceptual knowledge, including knowledge about how events unfold (see, e.g., He \& Arunachalam, 2017, for review), suggesting that by examining the developmental trajectory of (non)-culmination interpretations, we will obtain powerful insight into how children conceptualize events. During the preschool years, children map the linguistic forms of their language onto these event concepts, and must learn whether their language is like Hindi or English (see van Hout, 2018 for a recent review). Cross-linguistic developmental investigations will further reveal whether, and if so, when, children acquiring languages that differ along these lines diverge in their assignment of non-culmination interpretations to telic predicates.

\section{References}

Allopenna, Paul D., James S. Magnuson \& Michael K. Tanenhaus. 1998. Tracking the time course of spoken word recognition using eye movements: evidence for continuous mapping models. Journal of Memory and Language 38. 419-439.

https://doi.org/10.1006/jmla.1997.2558

Altmann, Gerry T. M. \& Yuki Kamide. 1999. Incremental interpretation at verbs: Restricting the domain of subsequent reference. Cognition 73(3). 247-264. https://doi.org/10.1016/S00100277(99)00059-1

Altmann, Gerry T. M. \& Yuki Kamide. 2007. The real-time mediation of visual attention by language and world knowledge: Linking anticipatory (and other) eye movements to linguistic processing. Journal of Memory and Language 57. 502-518.

https://doi.org/10.1016/j.jml.2006.12.004

Altmann, Gerry T. M., \& Yuki Kamide. 2009. Discourse-mediation of the mapping between language and the visual world: Eye movements and mental representation. Cognition, 111(1), 55-71. https://doi.org/10.1016/j.cognition.2008.12.005 
Altmann, Gerry T.M., \& Jelena Mirković. 2009. Incrementality and prediction in human sentence processing. Cognitive Science 33(4). 583-609. https://doi.org/10.1111/j.15516709.2009.01022.x.

Arunachalam, Sudha. 2017. Experimental approaches to understanding non-culmination in infants, children, and adults. Invited talk presented at TELIC 2017, Stuttgart, Germany.

Arunachalam, Sudha \& Anubha Kothari. 2011. An experimental study of Hindi and English perfective interpretation. Journal of South Asian Linguistics 4(1). 27-42.

Bar-El, Leora, Henry Davis \& Lisa Matthewson. 2005. On non-culminating accomplishments. Proceedings of the Annual Meeting of the North East Linguistics Society (NELS) 35. 87-102.

Barr, Dale J. 2008. Analyzing 'visual world' eyetracking data using multilevel logistic regression. Journal of Memory and Language 59(4). 457-474. https://doi.org/10.1016/j.jml.2007.09.002.

Bates, Douglas, Martin Maechler, Ben Bolker \& Steve Walker. 2015. Fitting linear mixed-effects models ising lme4. Journal of Statistical Software 67(1). 1-48. https://doi.org/10.18637/jss.v067.i01.

Bicknell, Klinton, Jeffrey L. Elman, Mary Hare, Ken McRae \& Marta Kutas. 2010. Effects of event knowledge in processing verbal arguments. Journal of Memory and Languag 63(4). 489-505. https://doi.org/10.1016/j.jml.2010.08.004.

Boersma, Paul \& David Weenink 2005. Praat: doing phonetics by computer [Computer program].

Bott, Oliver \& Anja Gattnar. 2015. The cross-linguistic processing of aspect-an eyetracking study on the time course of aspectual interpretation in Russian and German. Language, Cognition and Neuroscience 30(7). 877-898. https://doi.org/10.1080/23273798.2015.1029499.

Flecken, Monique, Christiane Stutterheim. \& Mary Carroll. 2014. Grammatical aspect influences motion event perception: Findings from a cross-linguistic non-verbal recognition task. Language and Cognition 6. 45-78. https://doi.org/10.1017/langcog.2013.2.

Foppolo, Francesca, Ciro Greco, Francesca Panzeri \& Maria Nella Carminati. 2018. The incremental processing of accomplishment predicates. Manuscript under review.

Gyarmathy, Zsófia \& Daniel Altshuler. 2017. Non-culmination by abduction. Linguistics 58(5). 1573-1411. https://doi.org/10.1515/ling-2020-0103.

He, Angela X. \& Sudha Arunachalam. 2017. How we conceptualize the ends of events (and what it might mean for verb acquisition). Paper presented at the $14^{\text {th }}$ International Congress for the Study of Child Language (IASCL), Lyon, France.

He, Angela X. \& Sudha Arunachalam. 2017. Word learning mechanisms. Wiley Interdisciplinary Reviews: Cognitive Science 8(4). e1435. https://doi.org/10.1002/wcs.1435.

Huang, Yi Ting, \& Jesse Snedeker. (2009). Online interpretation of scalar quantifiers: Insight into the semantics-pragmatics interface. Cognitive Psychology 58(3). 376-415.

Huang, Yi Ting, \& Jesse Snedeker. (2018). Some inferences still take time: Prosody, predictability, and the speed of scalar implicatures. Cognitive Psychology 102. 105-126.

Ikegami, Yoshihiko. 1985. 'Activity' - 'Accomplishment' - 'Achievement' - A language that can't say 'I burned it, but it didn't burn' and one that can. In A. Makkai \& A.K. Melby (eds.), Linguistics and philosophy: Essays in honor of Rulon S. Wells, 265-304. Amsterdam: John Benjamins. https://doi.org/10.1075/cilt.42.21ike. 
Kaiser, Elsi \& John Trueswell. 2004. The role of discourse context in the processing of a flexible word-order language. Cognition 94(2). 113-147. https://doi.org/10.1016/j.cognition.2004.01.002.

Koenig, Jean-Pierre \& Nuttannart Muansuwan. 2000. How to end without ever finishing: Thai semi-perfectivity. Journal of Semantics17(2). 147-182. https://doi.org/10.1093/jos/17.2.147.

Madden, Carol J. \& Rolf A. Zwaan. 2003. How does verb aspect constrain event representations? Memory \& Cognition 31(5). 663-672. https://doi.org/10.3758/BF03196106.

McRae, Ken \& Kazunaga Matsuki. 2009. People use their knowledge of common events to understand language, and do so as quickly as possible. Language and Linguistics Compass 3(6). 1417-1429. https://doi.org/10.1111/j.1749-818X.2009.00174.x.

Ogiela, Diane, Cristina Schmitt \& Michael Casby. 2014. Interpretation of verb phrase telicity: Sensitivity to verb-type and determiner-type. Journal of Speech, Language, and Hearing Research 57(3). 1-11. https://doi.org/10.1044/2013_JSLHR-L-12-0271.

Patt, Raymond, Laura Wagner \& Sudha Arunachalam. 2020. The development of a sense of an ending. In: Brown, Megan M. \& Kohut, Alexandra (Eds.), Proceedings of the $44^{\text {th }}$ annual Boston University Conference on Language Development (pp. 492-502). Somerville, MA: Cascadilla Press.

Pederson, Eric. 2007. Event realization in Tamil. In P. Brown \& M. Bowerman (eds.), Crosslinguistic perspectives on argument structure: Implications for learnability, 331-355.

New York: Lawrence Erlbaum.

Piñango, Maria M., Aaron Winnick, Rashad Ullah \& Edgar Zurif. 2006. Time-course of semantic composition: The case of aspectual coercion. Journal of Psycholinguistic Research 35(3). 233-244. https://doi.org/10.1007/s10936-006-9013-Z.

Todorova, Marina., Kathy Straub, William Badecker \& Robert Frank. 2000. Aspectual coercion and the online computation of sentential aspect. Proceedings of the Cognitive Science Society $1(1)$.

R Core Team (2013). R: A language and environment for statistical computing. R Foundation for Statistical Computing, Vienna, Austria.

Sakarias, Maria, \& M. Monique Flecken 2017. The result is in sight: Grammatical encoding of resultativity influences event perception and memory. Talk presented at the 30th Annual CUNY Conference on Human Sentence Processing. Cambridge, MA.

Singh, Mona. 1998. On the semantics of the perfective aspect. Natural Language Semantics 6(2). 171-199. https://doi.org/10.1023/A:1008208311200.

van Hout, Angeliek. 2018. On the acquisition of event culmination. In Sudha Arunachalam \& Kristen Syrett (eds.), Semantics in language acquisition, 96-121. Amsterdam \& Philadelphia: John Benjamins.

van Hout, Angeliek, Maria J. Arche, Hamida Demirdache, Isabel García del Real, Ainara García Sanz, Anna Gavarró, Lucía Gomez Marzo, Saar Hommes, Nina Kazanina \& Jinhong Liu. 2017. Agent control and the acquisition of event culmination in Basque, Dutch, English, Spanish and Mandarin. In Maria LaMendola \& Jennifer Scott (eds.), Proceedings of the 41st Annual Boston University Conference on Language Development, 323-332). Somerville, MA: Cascadilla Press. 